\title{
Leakage Resilient ElGamal Encryption
}

\author{
Eike Kiltz ${ }^{1}$ and Krzysztof Pietrzak ${ }^{2}$ \\ 1 Ruhr-Universität Bochum, Germany* \\ eike.kiltz@rub.de \\ 2 CWI, Amsterdam, The Netherlands \\ pietrzak@cwi.nl
}

\begin{abstract}
Blinding is a popular and well-known countermeasure to protect public-key cryptosystems against side-channel attacks. The high level idea is to randomize an exponentiation in order to prevent multiple measurements of the same operation on different data, as such measurements might allow the adversary to learn the secret exponent. Several variants of blinding have been proposed in the literature, using additive or multiplicative secret-sharing to blind either the base or the exponent. These countermeasures usually aim at preventing particular side-channel attacks (mostly power analysis) and come without any formal security guarantee.
\end{abstract}

In this work we investigate to which extend blinding can provide provable security against a general class of side-channel attacks. Surprisingly, it turns out that in the context of public-key encryption some blinding techniques are more suited than others. In particular, we consider a multiplicatively blinded version of ElGamal public-key encryption where

- we prove that the scheme, instantiated over bilinear groups of prime order $p$ (where $p-1$ is not smooth) is leakage resilient in the genericgroup model. Here we consider the model of chosen-ciphertext security in the presence of continuous leakage, i.e., the scheme remains chosen-ciphertext secure even if with every decryption query the adversary can learn a bounded amount (roughly $\log (p) / 2$ bits) of arbitrary, adversarially chosen information about the computation.

- we conjecture that the scheme, instantiated over arbitrary groups of prime order $p$ (where $p-1$ is not smooth) is leakage resilient.

Previous to this work no encryption scheme secure against continuous leakage was known. Constructing a scheme that can be proven secure in the standard model remains an interesting open problem.

\section{Introduction}

Side-channel attacks are cryptanalytic attacks against physical implementations of cryptosystems that exploit some kind of information leakage from the cryptodevice during execution. Traditional security notions (such as chosen-ciphertext security for encryption schemes) do not provide any security guarantee against such

\footnotetext{
* Part of the work conducted while the author was at CWI, Amsterdam.
} 
attacks, and many implementations of provably secure cryptosystems were broken by side-channel attacks exploiting side-channels such as running-time [36, electromagnetic radiation [4526, power consumption [38, fault detection 86] and many more (see, e.g., 46 43]).

Countermeasures against side channel attacks can either be algorithmic, or on the hardware level. In the latter case, one generally tries to build hardware that leaks as few information as possible (e.g., by shielding electromagnetic radiation.) Algorithmic countermeasures means that one designs algorithms, such that their mere description already provides security against side channel attacks. (E.g., one can protect against timing attacks by making sure that the running time of the algorithm is independent of the secret.) Traditionally, such algorithmic countermeasures (such as masking or blinding, cf. [43. for a list of relevant papers) are mostly ad-hoc in the sense that they defend against some specific and known attacks.

Leakage Resilient CRyptography. Recently, formal models were proposed where one does not assume any particular side-channel against which to protect, but only requires that potential side-channels are in some sense "resource bounded." In the model of leakage resilience [23], one considers adversaries which, on each invocation of the cryptographic primitive, can learn a bounded amount of arbitrary information about the secret internal state that was accessed during invocation. Since the overall amount of leaked information is unbounded (and may be much larger than the size of the secret state), this model is also often referred to as continuous leakage (e.g., [159]). As we will discuss below, this is in sharp contrast to the model of "memory leakage" (e.g., [2414316]) which has the inherent limitation that the amount of leaked information is a-priory bounded and therefore cannot exceed the size of the secret state.)

An implementation of a leakage resilient primitive will then be secure against every side-channel attack that fits our general model, i.e., as long as the amount of information that is leaked on each invocation is sufficiently bounded, and moreover the device adheres the "only computation leaks information" axiom from [40, which states that memory content that is not accessed during an invocation, does not leak. Security in this bounded leakage model hence means that the hardware implementation of the cryptosystem only has to be protected to fit the above model; once that is done, the proof provides security of the scheme. Using bounded leakage is inspired by the bounded retrieval model [13 20 19 |10|22 4 ] which in turn was inspired by the bounded-storage model [39 21 53 30].

So far most theoretical research has focused on preventing memory leakage 132019 1022 4 and the only known leakage resilient primitives (in our sense of security against continuous leakage) are stream-ciphers [23 44], digital signatures 25] and - in a weaker "non-adaptive" model — pseudorandom functions and permutations [18. Recently, general compilers have been proposed which turn any circuit into a leakage-resilient one 2833. Currently, these general compilers are just a proof of concept and too inefficient to be used in practice, relying on fully homomorphic encryption 33 or requiring one full encryption per gate [28]. 
In this paper, we address the problem of leakage resilient public-key encryption (PKE). The standard security notion for PKE is indistinguishability under a chosen plaintext attack (IND-CPA) or the stronger notion of indistinguishability under a chosen ciphertext attack (IND-CCA) 1

Modelling Leakage Resilience. Consider some cryptosystem CS, let $S_{0}$ denote its initial internal state and $S_{i}$ its state after the $i$ th invocation. On the $i$ th invocation of CS, the adversary chooses some input $X_{i}$ and gets $Y_{i}$ where $\left(S_{i+1}, Y_{i}\right) \leftarrow \operatorname{CS}\left(S_{i}, X_{i}\right)$

In the original definition of leakage resilience [23, the adversary gets the additional power to choose, besides the regular input $X_{i}$, some leakage function $f_{i}$ whose range is bounded to some fixed $\lambda \in \mathbb{N}$ bits with every query. After the $i$ th invocation she not only gets the regular output $Y_{i}$, but additionally the leakage $\Lambda_{i} \leftarrow f_{i}\left(S_{i}^{+}, R\right)$ where $R$ is the randomness that CS used during its computation, and $S_{i}^{+}$is the subset of the state $S_{i}$ that was accessed (i.e., read and/or written) during computation. Note that to be leakage resilient, a primitive must be stateful (i.e. $S_{i} \neq S_{i-1}$ ), as otherwise one can just leak the state $\lambda$ bits at a time.

In this paper we will use a more fine-grained notion of leakage resilience, where an invocation of CS (which will be a decryption query) is split in two phases, and those two phases leak individually. More precisely, the computation of a decryption can syntactically be split into two phases Dec1* and Dec2*, which are executed in a sequential order to decrypt the message. As in a CCA attack, the adversary can make decryption queries with respect to a ciphertext $C$, and can furthermore specify two (efficiently computable) leakage functions, $f$ and $g$, whose range is bounded by $\lambda$ bits. ( $\lambda$ is the leakage parameter.) In addition to the decryption of $C$ the adversary also obtains the output of $f$ and $g$ applied to all the inputs of Dec1* and Dec2*, respectively, including the algorithm's internal random coin tosses.

On Bounded Range And Domain. Summing up, leakage resilience considers attackers who, with every invocation, can adaptively choose a leakage function $f$ and then get the output of $f$ applied to the internal secret state (if the system is probabilistic also all internal coin tosses) of the cryptosystem. The function $f$ can be arbitrary, but is restricted in its input domain and range:

Bounded range: The range of $f$ is $\{0,1\}^{\lambda}$ for some parameter $\lambda \in \mathbb{N}$.

Bounded domain: $f$ gets as input only the secret state that is actually $a c$ cessed during this invocation.

A mathematical model of side-channel leakage is only useful if it captures (and thus implies security against) leakage that occurs in practice. As $f$ gets the

\footnotetext{
${ }^{1}$ In a CPA the adversary only gets the public-key and then has to distinguish the encryptions of two different messages. In a CCA 47 the adversary can also ask for decryptions of ciphertexts of her choice. We distinguish between CCA1 and the stronger CCA2 security, in the latter the adversary can make decryption queries also after she got the challenge ciphertext.
} 
same input as the cryptosystem CS, it can simulate the computation of CS on any conceivable hardware (e.g., all the values carried by wires on a computing circuit), and thus also compute any kind of leakage that might occur. Though, the restriction on bounded range might not allow $f$ to actually output the entire leakage, and the restriction on bounded domain might make it impossible to simulate leakage that depends on earlier invocations, we discuss this points below.

Bounded range. In practice, it seems hard to quantify how much information actual hardware (like a smart-card) actually leaks. In most side-channel attacks the adversary measures large amounts of data, e.g., an entire powerconsumption curve. So at a glance this assumption might seem unreasonable, but this is a bit overly pessimistic.

Even though side-channel leakage may contain lots of data, only a small fraction can actually be exploited in each measurement. The model of leakage resilience allows only for the leakage of a small number $\lambda$ of bits, but this leakage is "worst case" in the sense that the adversary may choose the leakage function which outputs the most useful information. Below we outline two ways in which this observation can be made precise. The first shows that side-channel attacks used in practice are captured by leakage resilience as they only exploit few bits of information from each actual measurement. The second is a relaxation of bounded leakage which can reasonably be assumed to be satisfied in practice.

Side-Channel Attacks Exploit Few Bits. Many side-channel attacks first measure large amounts of leakage $\Lambda_{1}, \Lambda_{2}, \ldots$ from every invocation, like a power consumption curve. Then, in a first step, each leakage $\Lambda_{i}$ is preprocessed in order to extract some "useful" information $\Lambda_{i}^{\prime}$ (this $\Lambda_{i}^{\prime}$ could, e.g., be a list of the most likely sub-keys.) The attack then proceeds by trying to recover the secret key from $\Lambda_{1}^{\prime}, \Lambda_{2}^{\prime}, \ldots$. Such attacks are covered by leakage resilience whenever the amount of extracted data $\left|\Lambda_{i}^{\prime}\right|$ is at most the amount of leakage $\lambda$ allowed per invocation.

Relaxing Bounded Range. By inspecting the proofs of our constructions (as well as the ones from [2344|25]), one sees that a restriction on the leakage functions is required which is considerably weaker than restricting the range to $\lambda$ bits: it is only required that the leakage $f\left(S^{+}\right)$does not decrease the HILL-pseudoentropy 315$]^{2}$ the adversary has about the active state $S^{+}$ by more than $\lambda$ bits. (More details will be given in the full version.) Thus, although it may be unreasonable to assume that no more than $\lambda$ bits leak per invocation of a smart-card, assuming that this leakage will only degrade the HILL-pseudoentropy by $\lambda$ bits seems much more realistic in practice.

Bounded domain. The bounded domain restriction is a very mild restriction. Unlike for bounded range, it is non-trivial to even imagine a remotely realistic side-channel attack which would break a scheme by not adhering to it. This

${ }^{2}$ HILL-pseudoentropy is a computational analogue of min-entropy. As for minentropy, $\lambda$ bits of information cannot decrease it (in expectation) by more than $\lambda$ bits. 
restriction (on how leakage functions are mathematically modeled) is implied by the "only computation leaks information" axiom (which states something about physical properties of devices) of [40]. But it also covers other practical attacks which do not satisfy this axiom. For example note that an adversary can learn any linear function $f(S)$ of the entire state $S$ (which is split in, say, two parts $S_{1}, S_{2}$ that are accessed individually) by specifying leakage functions $f_{1}, f_{2}$ such that $f_{1}(a)+f_{2}(b)=f(a, b)$ (the adversary can ask to learn $f_{1}\left(S_{1}\right)$ and $f_{2}\left(S_{2}\right)$ as $S_{1}$ and $S_{2}$ are accessed respectively, and then compute $f(S)$ locally.) This simple observation already shows that claims made in the literature arguing that the bounded range \& domain restrictions do not cover attacks like "cold-boot attacks" [29] or static leakage (as claimed in [51]) are not well-founded 3 As argued by Dziembowski 4 this restriction not only covers all linear function $f(a, b)=f_{1}(a)+f_{2}(b)$, but in fact any function $f(a, b)$ which has a communication complexity of at most $\lambda$. A good candidate for an actual leakage function that does invalidate this assumption 5 is the inner product $f(a, b)=\sum_{i} a_{i} \cdot b_{i} \bmod 2$ which has linear communication complexity.

\subsection{ElGamal Encryption}

The ElGamal encryption scheme 24 over a cyclic group $\mathbb{G}$ of prime order $p$ works as follows. The public key consists of a generator $g$ of $\mathbb{G}$ and $X=g^{x}$, where $x \in \mathbb{Z}_{p}$ is the secret key. Encryption defines the ciphertext as $C=g^{r}$ and uses the symmetric key $K=X^{r}$ to blind the message. Decryption reconstructs the key by computing $K=C^{x}$. In its hybrid version, ElGamal encryption is contained in many standard bodies (e.g., [483250]) and it is (using the name Elliptic Curve Integrated Encryption System, "ECIES") commonly considered to be the standard method to encrypt over elliptic curves. At this point it may be instructive to see why the ElGamal encryption scheme is not leakage resilient. An adversary, in the $i$ th decryption query, can specify a leakage function that outputs the $i$-th bit of the secret key $x$. Therefore, after $q=|x|$ queries to the leakage oracle the entire secret key can be reconstructed. As we already pointed out, the inherent reason why the above attack works is that decryption is stateless.

Let's first look at a straight forward (but unsuccessful) attempt to make the ElGamal scheme leakage resilient. To this end we make decryption stateful and

${ }^{3}$ In the above argument we implicitly assumed that ultimately the entire secret state will be touched, although this seems obvious (after all, why would one save a secret state if it's not supposed to be ever read), the tokens used in the construction of onetime programs 27] are an example where exactly this happens. For such primitives obeying the "only computation leaks information" axiom in its original physical sense is necessary.

4 At the workshop "Provable security against physical attacks", February 2010, Leiden.

${ }^{5}$ And thus might be used to construct an actual real world counterexample where the security of an implementation gets broken because the bounded domain restriction is invalidated. 
split it into two parts Dec1* and Dec2*. The secret key is additively shared into $x=\sigma_{0}+\sigma_{0}^{\prime}$ by setting $\sigma_{0}=x-r_{0}$ and $\sigma_{0}^{\prime}=x+r_{0}$. Decryption works as follows. The first part Dec1 ${ }^{*}$ computes $\sigma_{i}=\sigma_{i-1}+r_{i} \bmod p, K^{\prime}=C^{\sigma_{i}}$ and passes $K^{\prime}$ as input to the second part. Dec2* computes $\sigma_{i}^{\prime}=\sigma_{i-1}^{\prime}-r_{i} \bmod p$ and then $K=K^{\prime} \cdot C^{\sigma_{i}^{\prime}}$. Note that the state information is randomly re-shared subject to $\sigma_{i}+\sigma_{i}^{\prime}=x$. However, this scheme is not leakage resilient since an attacker can adaptively learn certain bits of $\sigma_{i}=x+R_{i}$ and $\sigma_{i}^{\prime}=x-R_{i}$ (where $\left.R_{i}=\sum_{j=0}^{i} r_{j}\right)$ that enable him to fully reconstruct the secret key $x$

\subsection{Our Results}

Conjectured leakage Resilient ElGamal encryption. We consider a practical randomization method to make the ElGamal PKE scheme (or one if its standardized hybrid variants) leakage resilient under chosen-ciphertext attacks in the above sense. In the context of leakage resilience this method (or variants thereof) were already proposed in [12/37/52. The central idea is to use multiplicative secret sharing to share the secret key $x$, i.e., $x$ is shared as $\sigma_{i}=x R_{i}^{-1} \bmod p$ and $\sigma_{i}^{\prime}=R_{i} \bmod p$, for some random $R_{i} \in \mathbb{Z}_{p}^{*}$. More precisely, the first part of decryption computes $\sigma_{i}=\sigma_{i-1} r_{i}^{-1} \bmod p$ and $K^{\prime}=C^{\sigma_{i}}$. The second part computes $\sigma_{i}^{\prime}=\sigma_{i-1}^{\prime} r_{i} \bmod p$ and then $K=K^{\prime \sigma_{i}^{\prime}}$. Again note that the state information is randomly reshared subject to $\sigma_{i} \cdot \sigma_{i}^{\prime}=x$. We remark that our method does not modify ElGamal's encryption algorithm, it only modifies the way ciphertexts are decrypted. In particular, public-keys and ciphertexts are the same as in ElGamal encryption and therefore our method offers an attractive way to update existing ElGamal-based systems with algorithmic security against side-channel attacks. Unfortunately, we are not able to prove that the above method is provable leakage resilient and therefore we can only state the scheme's security as a conjecture.

Provable leakage Resilient ElGamal encryption. We also propose to apply multiplicative secret sharing to the ElGamal encryption scheme instantiated over bilinear groups. Our main theorem (Theorem1) states that this scheme is leakage resilient against CCA1 attack in the generic group model. The key observation is that the secret key is a group element $X$ and decryption performs a pairing operation with $X$ as one fixed base. This allows us to multiplicatively share the secret key as a group element, i.e., $X=\sigma_{i} \cdot \sigma_{i}^{\prime} \in \mathbb{G}$. Intuitively, we use the fact that in the generic group model some bits of the representation of $\sigma_{i}$ and $\sigma_{i}^{\prime}$ essentially look random and therefore are useless to the leakage adversary. To formally prove this intuition, however, turns out to be surprisingly difficult.

We also mention that a proof in the generic group model has its obvious weaknesses. (See, e.g., 35].) In particular in connection with side channel attacks

\footnotetext{
${ }^{6}$ Since $x=\sigma_{i}+\sigma_{i}^{\prime} \bmod p$, the first $t \approx \lambda$ least significant bits of $x$ can be computed as $\left(\sigma_{i} \bmod 2^{t}\right)+\left(\sigma_{i}^{\prime} \bmod 2^{t}\right) \bmod 2^{t}$, minus an additive factor $p \bmod 2^{t}$ in case there is an overflow $\bmod p$. (The latter can be checked from the high order bits of $\sigma_{i}$ and $\sigma_{i}^{\prime}$.) This process can be iterated to learn the entire secret key.
} 
the generic group model may "abstract away" too much important information an adversary may obtain in a real implementation of the scheme. This should be taken into account when interpreting our formal security statement. However, our result seems to be the first PKE scheme that is provably leakage resilient. Furthermore, the scheme is very practical. Another possible interpretation of our result is that when protecting the exponentiation function against (a large class of) side-channel attacks, multiplicative secret sharing techniques seem more suitable than additive ones.

Leakage Resilient Exponentiation and Pairing Operation. Speaking more generally, our above mentioned methods how to secure ElGamal against side-channel attacks show that one can possibly make discrete exponentiation and a pairing operation leakage resilient. Let $\mathbb{G}$ be a group of prime order $p$ and $g$ be a generator of $\mathbb{G}$. In discrete exponentiation one wants to take public group elements $Y_{i}$ to some fixed secret power $x$ (which is only leaked through $\left.g^{x}\right)$. We propose to share $x$ as $x=x^{\prime} \cdot x^{\prime \prime} \bmod p$ and compute the values $K_{i}=Y_{i}^{x}$ in two iterative steps as $K_{i}^{\prime}=Y_{i}^{x^{\prime}}$ followed by $K_{i}=\left(K_{i}^{\prime}\right)^{x^{\prime \prime}}$. After each such computation $x^{\prime}$ and $x^{\prime \prime}$ get randomly reshared subject to $x=x^{\prime} \cdot x^{\prime \prime} \bmod p$. In a pairing operation one is given public group elements $Y_{i}$ and want to compute $e\left(Y_{i}, X\right)$, for some fixed secret group element $X$ (which is only leaked though $e(g, X))$. Here $e: \mathbb{G} \times \mathbb{G} \rightarrow \mathbb{G}_{T}$ is a bilinear pairing. Again we propose to share $X$ as $X=X^{\prime} \cdot X^{\prime \prime} \in \mathbb{G}$ and compute the values $K_{i}=e\left(Y_{i}, X\right)$ in three iterative steps as $K_{i}^{\prime}=e\left(Y_{i}, X^{\prime}\right), K_{i}^{\prime \prime}=e\left(Y_{i}, X^{\prime \prime}\right)$, and $K_{i}=K_{i}^{\prime} \cdot K_{i}^{\prime \prime} \in \mathbb{G}_{T}$, followed by a resharing of $X=X^{\prime} \cdot X^{\prime \prime} \in \mathbb{G}$. Our main result (Theorem 1) shows that our method to perform a pairing operation is provable leakage resilient in the generic group model.

Difficulty to PRove leakAge Resilience against CCA2 ATtACKs. It is well known that the ElGamal encryption scheme, where the key $K$ is hashed and the one-time pad is replaced with a chosen-ciphertext secure symmetric cipher, is secure against CCA2 attacks [1. We remark that this scheme is not leakage resilient against CCA2 attack since an adversary can adaptively obtain some bits about the unhashed symmetric key of the challenge ciphertext. Indeed, building a PKE scheme that is (provably) leakage resilient against CCA2 attacks remains a challenging open problem.

\subsection{Related Work}

In the hardware community the usefulness of secret-sharing in the context of sidechannel countermeasures is well known. In particular, secret-sharing has been proposed as a countermeasure against "differential power analysis attacks" for exponentiation algorithms in [111212 37/52], but without any formal analysis.

Most works on side-channel countermeasures, including the ones just mentioned, consider countermeasures against particular side-channel attacks. Micali and Reyzin [40] in their work on "physically observable cryptography" proposed an influential theoretical framework to capture side-channel attacks on a more general level. 
Besides leakage resilience, there are several other models that consider cryptosystems which remain secure even if a function $f(s k)$ (chosen by the adversary from a very broad class of functions) of the secret key $s k$ is leaked. We shortly mention these models below. The main difference to leakage resilience is that those models consider stateless cryptosystems, and thus cannot tolerate any kind of "continuous" leakage (an exception is the very recent work on "continuous memory attacks.") On the other hand, the leakage function in those works gets the entire state as input, and not just the part of the state that was accessed.

Memory AtTacks. Akavia et al. 2] introduce the model of "security against memory attacks," where one requires that the scheme remains secure even if a function $f(s k)$ of the secret $s k$ is leaked once, where the only restriction on $f(\cdot)$ one makes is its bounded output length. (Clearly the bound must satisfy $|f(s k)| \ll|s k|$. This model is a restricted version of the BRM model discussed below.) 241] construct public-key encryption schemes in this model, Katz and Vaikuntanathan 34] constructs digital signatures.

Bounded Retrieval Model. The bounded retrieval model (BRM) [13 19 20,10 22 4] is a generalization of the previous model, where one requires that the secret key can be made huge, while the scheme still remains efficient. Such schemes can provide security against malware like viruses or Trojans, which temporarily take control over a computer, but do not have enough "bandwidth" to leak the entire artificially huge key. Most works on intrusion resilient crypto consider symmetric primitives, but after the first success in constructing publickey cryptosystems secure against memory attacks (mentioned above), Alwen et al. achieved public-key crypto also in the BRM model. In particular authentication and signature schemes [4] and public-key encryption [3].

Auxiliary InPut. Dodis et al. construct symmetric [17] and public-key [14] encryption schemes in a model where the range of $f(\cdot)$ may be unbounded, but one only requires that it is hard to recover $s k$ from $f(s k)$. (i.e. any polynomial time adversary should output $s k$ with exponentially small probability.)

Continuous Memory Attacks. Very recently, Dodis, Haralambiev, Lopez-Alt, and Wichs 15] and Brakerski, Kalai, Katz and Vaikuntanathan 9] introduce the model of "continuous memory attacks." This model generalizes the notion of memory attacks. Also here the adversary can learn a bounded amount, $\lambda$ bits say, of leakage about the (entire) secret key. But now there's an additional "refresh" procedure which takes the secret key $s k$ and outputs a new secret key $s k^{\prime}$. The adversary can learn $\lambda$ bits (where $\lambda$ is $c|s k|$ for some constant $c>0$ ) in-between any two refresh phases, but the refreshing itself has to be completely leak-free [15] or leak at most a logarithmic number of bits [9]. Remarkably, in this model [15] construct authentication and signature schemes, 9] obtain get public-key encryption. Both papers work in the standard model, the underlying assumption in both papers is the linear assumption over bilinear groups. The models of leakage resilience and continuous memory attacks are incomparable: leakage resilience assumes "only computation leaks" whereas continuous memory attacks need an (almost) leakfree refresh phase. As mentioned, the constructions [15.9] are proven secure in the 
standard model, whereas we use a strong idealized model. On the positive side, our scheme is very efficient (only about two times slower than standard ElGamal) whereas, e.g., 9 needs a constant number of pairings to encrypt a single bit.

\section{Definitions}

If $A$ is a deterministic algorithm we write $y \leftarrow A(x)$ to denote that $A$ outputs $y$ on input $x$. If $\mathrm{A}$ is randomized we write $y \stackrel{*}{\leftarrow} \mathrm{A}(x)$ or, $y \stackrel{r}{\leftarrow} \mathrm{A}(x)$ if we want to make the randomness $r$ used by the algorithm explicit (for future reference).

Key Encapsulation Mechanisms. A key encapsulation mechanism (KEM) is defined similarly to a public-key encryption scheme, except that the encryption algorithm (called encapsulation) does not take any input, but rather outputs the encryption of a random key $K$, which then can be used with as a key in any symmetric encryption scheme to encrypt the actual message.

Formally, a key-encapsulation mechanism KEM consists of three algorithms KG, Enc, Dec. KG : $\{0,1\}^{*} \rightarrow \mathcal{P K} \times \mathcal{S K}$ is the probabilistic key-generation algorithm, which on input a security parameter $\kappa$ outputs a public/secret-key pair. The probabilistic encapsulation algorithm Enc $: \mathcal{P} \mathcal{K} \rightarrow \mathcal{K} \times \mathcal{C}$ and decapsulation algorithm Dec $: \mathcal{S K} \times \mathcal{C} \rightarrow \mathcal{K} \cup \perp$ satisfy the following correctness property for all $\kappa$

$$
\operatorname{Pr}\left[K=K^{\prime} \mid(p k, s k) \stackrel{*}{\leftarrow} \mathrm{KG}(\kappa) ;(C, K) \stackrel{*}{\leftarrow} \operatorname{Enc}(p k) ; K^{\prime} \leftarrow \operatorname{Dec}(s k, C)\right]=1
$$

The CCA1 security (aka. security against lunchtime attacks) of a key-encapsulation mechanism KEM is defined by the following experiment.

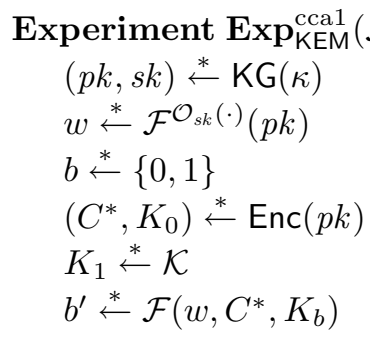

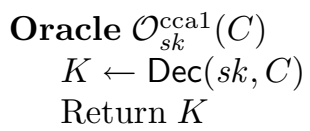

Let $\mu$ denote the probability that $b=b^{\prime}$ in the above experiment, then we define the advantage of $\mathcal{F}$ as $\operatorname{Adv}_{\text {KEM }}^{\text {cca1 }}(\mathcal{F}, \kappa)=2|1 / 2-\mu|$. In CCA2 security, the adversary is additionally allowed to query the decryption oracle in its second (guess) stage.

Stateful key encapsulation and leakage resilience. To formally define our notion of leakage resilience we consider stateful key encapsulation mechanisms $\mathrm{KEM}^{*}=\left(\mathrm{KG}^{*}, \mathrm{Enc}^{*}, \mathrm{Dec}^{*}\right.$, Dec2 $\left.{ }^{*}\right)$ in which decapsulation is stateful and can formally split into two sequential stages $\operatorname{Dec}=\left(\operatorname{Dec}{ }^{*}, \mathrm{Dec}{ }^{*}\right)$. The input/output behavior will stay exactly the same as in a standard KEM. 
More formally, the key generation algorithm $\mathrm{KG}^{*}(\kappa)$ generates a public key and and two initial states, $\sigma_{0}$ and $\sigma_{0}^{\prime}$. Intuitively, the states shares the secret key of the scheme and will be used by the stateful decapsulation algorithms Dec1*, Dec2* .

On the $i$ th invocation of decapsulation, the decapsulated key $K_{i}$ is computed as follows

$$
\left(\sigma_{i}, w_{i}\right) \stackrel{r_{i}}{\leftarrow} \operatorname{Dec}^{*}\left(\sigma_{i-1}, C_{i}\right) ;\left(\sigma_{i}^{\prime}, K_{i}\right) \stackrel{r_{i}^{\prime}}{\leftarrow} \operatorname{Dec}^{*}\left(\sigma_{i-1}^{\prime}, w_{i}\right)
$$

Here $r_{i}$ and $r_{i}^{\prime}$ is the explicit randomness of the two randomized algorithms, $\sigma_{i}$ and $\sigma_{i}^{\prime}$ are the updated states and $w_{i}$ is some state information that is passed from Dec1* to Dec2*.

We now define leakage resilience. Let $\lambda \in \mathbb{N}$ be some leakage parameter. We will consider attacks, where the adversary can not only query its oracle for the decapsulated values $K_{i}=\operatorname{Dec}\left(s k, C_{i}\right)$, but additionally gets leakage from the computation of those values. That is, in the security experiment the adversary can, additionally to the input $C_{i}$, specify two efficiently computable leakage functions $f_{i}, g_{i}$ with bounded range $\{0,1\}^{\lambda}$, and additionally to the regular output $K_{i}$ also gets $\Lambda_{i}, \Lambda_{i}^{\prime}$ computed as

$$
\Lambda_{i}=f_{i}\left(\sigma_{i-1}, r_{i}\right) ; \Lambda_{i}^{\prime}=g_{i}\left(\sigma_{i-1}^{\prime}, w_{i}, r_{i}^{\prime}\right),
$$

where the notation is as in (11). So the functions $f_{i}, g_{i}$ get as input exactly the same data as Dec1*/Dec2*7 We define the CCLA1 (chosen ciphertext with leakage attack) security of KEM by the experiment below. (Note that now we not only have to specify the security parameter $k$, but also a leakage bound $\lambda$.)

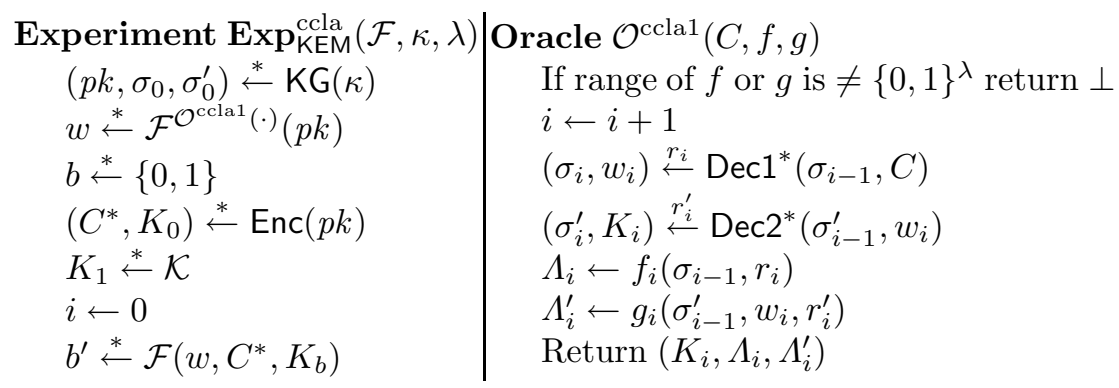

Let $\mu$ denote the probability that $b=b^{\prime}$ in the above experiment, then we define the advantage of $\mathcal{F}$ as $\operatorname{Adv}_{\text {KEM }}^{\text {ccla }}(\mathcal{F}, \kappa, \lambda)=2|1 / 2-\mu|$.

It is well-known that a CCA1 secure KEM plus a one-time secure symmetric cipher (such as a one-time pad) yields a CCA1-secure PKE scheme. For trivial reasons the same statement is also true for CCLA1 secure KEMs so for our purpose it is sufficient to build a CCLA1 secure KEM. On the other hand we remark that the respective composition theorem is wrong in general for CCLA2 secure KEMs.

\footnotetext{
${ }^{7}$ Note that $C_{i}$ need not be explicitly given to $f_{i}$ as the adversary chooses $f_{i}$ and $C_{i}$ together, and thus can "hard-code" $C_{i}$ into $f_{i}$.
} 
That is, a CCLA2 secure KEM and a CCA secure DEM will in general not yield a CCLA2 secure PKE scheme 8

Bilinear Groups. We assume the existence of a bilinear group generator BGen which is a randomized algorithm that outputs a bilinear group $\mathbb{P} G=\left(\mathbb{G}_{1} \mathbb{G}_{T}, g\right.$, $e, p)$ such that the following properties hold.

1. $\mathbb{G}$ and $\mathbb{G}_{T}$ are (multiplicative) cyclic groups of prime order $p$.

2. $g$ is a generator of $\mathbb{G}$.

3. $e$ is a bilinear map $e: \mathbb{G} \times \mathbb{G} \rightarrow \mathbb{G}_{T}$ which is

(a) bilinear: for all $u, v \in \mathbb{G}$ and $a, b \in \mathbb{Z}, e\left(u^{a}, v^{b}\right)=e(u, v)^{a b}$.

(b) non-degenerate: $e(g, g) \neq 1$.

We say that $\mathbb{G}$ is a bilinear group if there exists a group $\mathbb{G}_{T}$ and a bilinear map $e: \mathbb{G} \times \mathbb{G} \rightarrow \mathbb{G}_{T}$ as above, where $e$ and the group action in $\mathbb{G}$ and $\mathbb{G}_{T}$ can be computed efficiently. We will use $\circ$ and $\star$ for the group operation in $\mathbb{G}$ and $\mathbb{G}_{T}$ respectively.

Generic Bilinear Groups. In the generic group model 42 49] one encodes the group elements by unique, randomly chosen strings. This enforces that the only property which can be tested by an adversary is equality.

In the generic bilinear model (GBG ) [7] the encoding is given by randomly chosen injective functions $\xi: \mathbb{Z}_{p} \rightarrow \Xi$ and $\xi_{T}: \mathbb{Z}_{p} \rightarrow \Xi_{T}$ which give the representations of the elements in the base and target group respectively (w.l.o.g. we will assume that $\Xi \cap \Xi_{T}=\emptyset$ ). The group operation and the bilinear map are performed by three public oracles $\mathcal{O}, \mathcal{O}_{T}, \mathcal{O}_{e}$, where for any $a, b \in \mathbb{Z}_{p}$

$-\mathcal{O}(\xi(a), \xi(b)) \rightarrow \xi(a+b \bmod p)$

(group operation on base group).

$-\mathcal{O}_{T}\left(\xi_{T}(a), \xi_{T}(b)\right) \rightarrow \xi_{T}(a+b \bmod p)$

$-\mathcal{O}_{e}(\xi(a), \xi(b)) \rightarrow \xi_{T}(a \cdot b \bmod p)$

(group operation on target group). All oracles output $\perp$ when queried on an input outside of their domain. For a fixed generator $g$ of $\mathbb{G}$ and $g_{T} \stackrel{\text { def }}{=} e(g, g)$, one can think of $\xi(a)$ as an encoding of $g^{a}$, $\xi_{T}(a)$ as an encoding of $g_{T}^{a}$ and $\xi_{e}(a, b)$ as an encoding of $g_{T}^{a \cdot b}=e\left(g^{a}, g^{b}\right)$. Of course one also must provide some means of computing the group representation $\xi(a)$ or $\xi_{T}(a)$ for any $a \in \mathbb{Z}_{p}$, say by providing oracles to do so. We can get away without additional oracles, by providing $\xi(1)$ and observing that then $\xi(a)$ can be computed making $\leq 2 \log p$ queries to $\mathcal{O}$ (by square and multiply). $\xi_{T}(1)$ (and thus any $\left.\xi_{T}(a)\right)$ can be computed by $\xi_{T}(1) \leftarrow \mathcal{O}_{e}(\xi(1), \xi(1))$.

\section{Leakage Resilient ElGamal Encryption}

In this section we present a general method to secure ElGamal encryption against leakage attacks. First, we present a modification of the standard ElGamal cryptosystem over any cyclic group of prime order. Unfortunately, we are not able to

\footnotetext{
${ }^{8}$ An attacker may make a number of decryption queries only modifying the symmetric part of the challenge ciphertext. The decryption algorithm (internally) uses the challenge symmetric key that can be learned (bit-by-bit) through the leakage function.
} 
formally prove the leakage resilience of this scheme so we state its security as a conjecture. Next, we move to the ElGamal scheme over Bilinear Groups. Here we are able to prove that our method leads to a leakage resilient public-key encryption scheme (in the sense of CCLA1) in the generic group model.

\subsection{ElGamal Key Encapsulation}

Let $G e n$ be a randomized algorithm that outputs a cyclic group $\mathbb{G}$ of order $p$ where $p$ is a strong prime. The ElGamal key-encapsulation mechanism $\mathrm{EG}=\left(\mathrm{KG}_{\mathrm{EG}}\right.$, Enc $_{E G}$, Dec $_{E G}$ ) is defined as follows.

- $\mathrm{KG}_{\mathrm{EG}}(\kappa)$ : Compute $(\mathbb{G}, p) \stackrel{*}{\leftarrow} \mathrm{Gen}(\kappa)$ and choose random $g \stackrel{*}{\leftarrow} \mathbb{G}$ and random $x \stackrel{*}{\leftarrow} \mathbb{Z}_{p}$. Set $X=g^{x}$. The public key is $p k=(\mathbb{G}, p, X)$ and the secret key is $s k=x$.

- $\operatorname{Enc}_{\mathrm{EG}}(p k)$ : choose random $r \stackrel{*}{\leftarrow} \mathbb{Z}_{p}$. Set $C \leftarrow g^{r} \in \mathbb{G}$ and $K \leftarrow X^{r} \in \mathbb{G}$. The ciphertext is $C$ and the key is $K$.

- $\operatorname{Dec}_{\mathrm{EG}}(s k, C)$ : Compute the key as $K=C^{x} \in \mathbb{G}$.

As mentioned in the introduction, EG (or any other stateless scheme) cannot be leakage resilient since in the CCLA1 experiment an adversary can simply obtain the successive bits of the secret key $x$.

We will now describe a leakage resilient stateful key encapsulation mechanism $\mathrm{EG}^{*}=\left(\mathrm{KG}_{\mathrm{EG}}^{*}, \mathrm{Enc}_{\mathrm{EG}}^{*}\right.$, Dec1 $1_{\mathrm{EG}}^{*}$, Dec2 $\left.{ }_{\mathrm{EG}}^{*}\right)$, which is derived from $\mathrm{EG}$. As described in Section 2, the decapsulation algorithm is stateful and split in two parts.

$-\mathrm{KG}_{\mathrm{EG}}^{*}(\kappa)$ : Run $(s k, p k) \stackrel{*}{\leftarrow} \mathrm{KG}_{\mathrm{EG}}(\kappa)$. (Recall that $s k=x$ and $p k=(\mathbb{G}, p, X=$ $\left.g^{x}\right)$.) Choose random $\sigma_{0} \stackrel{*}{\leftarrow} \mathbb{Z}_{p}^{*}$ and set $\sigma_{0}^{\prime}=x \sigma_{0}^{-1} \bmod p$. The public key is $p k$ and the two secret states are $\sigma_{0}$ and $\sigma_{0}^{\prime}$.

- $\operatorname{Enc}_{\mathrm{EG}}^{*}(p k)$ : the same as $\operatorname{Enc}_{\mathrm{EG}}(p k)$.

- $\operatorname{Dec}_{\mathrm{EG}}^{*}\left(\sigma_{i-1}, C\right)$ : choose random $r_{i} \stackrel{*}{\leftarrow} \mathbb{Z}_{p}^{*}$, set $\sigma_{i}=\sigma_{i-1} r_{i}^{-1} \bmod p, K^{\prime}=C^{\sigma_{i}}$ and return $\left(r_{i}, K^{\prime}\right)$.

$-\operatorname{Dec} 2_{\mathrm{EG}}^{*}\left(\sigma_{i-1}^{\prime},\left(r_{i}, K^{\prime}\right)\right)$ : set $\sigma_{i}^{\prime}=\sigma_{i-1}^{\prime} r_{i}^{-1} \bmod p$, and $K=K^{\prime \sigma_{i}^{\prime}}$. The symmetric key is $K$ and the updated state information is $\sigma_{i}$ and $\sigma_{i}^{\prime}$.

We cannot formally prove CCLA1 security of the scheme so we have to resort to the following conjecture.

Conjecture 1. EG ${ }^{*}$ is CCLA1 secure if $p-1$ has a large prime factor (say, $p-1=2 p^{\prime}$ for a prime $\left.p^{\prime}\right) 9$

${ }^{9}$ The reason we require $p$ to be not smooth is to prevent the leakage functions to possibly compute discrete logarithms in $\mathbb{Z}_{p-1}$, as otherwise the multiplicative sharing $\sigma, \sigma^{\prime}$ (where $\sigma \cdot \sigma^{\prime}=x$ ) can be efficiently turned into an additive sharing (of the discrete $\log$ of the secret key) $\sigma=h^{\Sigma}, \sigma^{\prime}=h^{\Sigma^{\prime}}$ where $x=h^{X}$ and $X=\Sigma+\Sigma^{\prime}$. As described in Section 1.1 an additive sharing cannot give a leakage resilient scheme. The above also hints the inherent difficulty of proving this conjecture. Let us mention that already in [37] it is suggested to use a prime $\mathrm{p}$ where $(\mathrm{p}-1) / 2$ is prime in a very similar context. Our result can be seen as a formal justification for this choice. 
One can furthermore make ElGamal key-encapsulation CCA2 secure (without leakage) under the strong Diffie-Hellman assumption in the random oracle model by hashing the symmetric key symmetric key $K[1$. This Hashed ElGamal scheme is contained in many standard bodies, e.g. 4832 50. Hashing the symmetric key clearly does not affect its CCLA1 security and therefore Hashed ElGamal is CCLA1 and CCA2 secure.

However, as we will explain now, in our leakage resilience setting hashing $K$ will not make the scheme CCLA2 secure. The (unhashed) EG scheme is not CCA2 secure since it is malleable. (An adversary, given the challenge ciphertext $C$ (enciphering a key $K$ ), can ask for a decryption of $C^{2} \neq C$ to obtain $K^{2}$ from which it can reconstruct $K$.) Without considering leakage, hashing the key prevents this attack as now the adversary only sees a hashed key $\mathcal{H}\left(K^{2}\right)$. Unfortunately, in the leakage setting hashing will not help at all because the adversary can specify a leakage function which outputs $\lambda$ bits of the unhashed key $K^{2}$. By asking for the decryption of the same ciphertext $C^{2}$ several times, leaking $\lambda$ different bits of $K^{2}$ on each invocation, will ultimately reveal the entire $K^{2}$.

\subsection{Bilinear ElGamal Key Encapsulation}

The Bilinear ElGamal key-encapsulation mechanism $\mathrm{BEG}=\left(\mathrm{KG}_{\mathrm{BEG}}, \mathrm{Enc}_{\mathrm{BEG}}, \mathrm{Dec}_{\mathrm{BEG}}\right)$ is defined as follows.

- $\mathrm{KG}_{\mathrm{BEG}}(\kappa)$ : Compute $\mathbb{P} \mathbb{G}=\left(\mathbb{G}, \mathbb{G}_{T}, p, e\right) \stackrel{*}{\leftarrow} \mathrm{BGen}(\kappa)$ and choose random $g \stackrel{*}{\leftarrow}$ $\mathbb{G}$ and random $x \stackrel{*}{\leftarrow} \mathbb{Z}_{p}$. Set $X=g^{x}$ and $X_{T}=e(g, g)^{x}$. The public key is $p k=\left(\mathbb{P G}, g, X_{T}\right)$ and the secret key is $s k=X$.

- $\operatorname{Enc}_{\mathrm{BEG}}(p k)$ : choose random $r \stackrel{*}{\leftarrow} \mathbb{Z}_{p}$. Set $C \leftarrow g^{r} \in \mathbb{G}$ and $K \leftarrow X_{T}^{r} \in \mathbb{G}_{T}$. The ciphertext is $C$ and the key is $K$.

- $\operatorname{Dec}_{\mathrm{BEG}}(s k, C)$ : Compute the key as $K=e(C, X) \in \mathbb{G}_{T}$.

Note that correctness follows from the bilinear property $X_{T}^{r}=e(g, g)^{x r}=$ $e\left(g^{r}, g^{x}\right)=e\left(g^{r}, X\right)$.

We will now describe a leakage resilient key encapsulation $\mathrm{BEG}^{*}=\left(\mathrm{KG}_{\mathrm{BEG}}^{*}, \mathrm{Enc}_{\mathrm{BEG}}^{*}, \operatorname{Dec}_{\mathrm{BEG}}^{*}, \mathrm{Dec}_{\mathrm{BEG}}^{*}\right)$, which is derived from BEG.

$-\mathrm{KG}_{\mathrm{BEG}}^{*}(\kappa)$ : Run $(s k, p k) \stackrel{*}{\leftarrow} \mathrm{KG}_{\mathrm{BEG}}(\kappa)$. (Recall that $s k=X=g^{x}$ and $p k=$ $\left(\mathbb{P} G, g, X_{T}=e(g, g)^{x}\right.$.) Choose random $r_{0} \stackrel{*}{\leftarrow} \mathbb{Z}_{p}^{*}$ and set $\sigma_{0} \leftarrow g^{r_{0}}, \sigma_{0}^{\prime} \leftarrow$ $g^{x-r_{0}}$. The public key is $p k$ and the secret states are $\sigma_{0}, \sigma_{0}^{\prime}$.

- $\operatorname{Enc}_{\mathrm{BEG}}^{*}(p k)$ : the same as $\operatorname{Enc}_{\mathrm{BEG}}(p k)$.

- $\operatorname{Dec}_{\mathrm{BEG}}^{*}\left(\sigma_{i-1}, C\right)$ : choose random $r_{i} \stackrel{*}{\leftarrow} \mathbb{Z}_{p}$, set $\sigma_{i} \leftarrow \sigma_{i-1} \circ g^{r_{i}}, K^{\prime} \leftarrow e\left(\sigma_{i}, C\right)$ and return $\left(r_{i}, K^{\prime}\right)$.

$-\operatorname{Dec} 2_{\mathrm{BEG}}^{*}\left(\sigma_{i-1}^{\prime},\left(r_{i}, K^{\prime}\right)\right)$ : set $\sigma_{i}^{\prime} \leftarrow \sigma_{i-1}^{\prime} \circ g^{-r_{i}}$ and $K^{\prime \prime} \leftarrow e\left(\sigma_{i}^{\prime}, C\right)$. The symmetric key is $K \leftarrow K^{\prime} \star K^{\prime \prime} \in \mathbb{G}_{T}$.

Note that for every $i, R_{i} \stackrel{\text { def }}{=} \sum_{j=0}^{i} r_{j}$, we have $\sigma_{i} \circ \sigma_{i}^{\prime}=g^{R_{i}} \circ g^{x-R_{i}}=g^{x}$, so the $\sigma_{i}, \sigma_{i}^{\prime}$ are a secret sharing of the secret key. 
Theorem 1. In the bilinear generic group model the scheme $\mathrm{BEG}^{*}$ is CCLA1 secure: the advantage of a q-query adversary who gets $\lambda$ bits of leakage per invocation of Dec1 $1_{\mathrm{BEG}}^{*}$ and Dec2 ${ }_{\mathrm{BEG}}^{*}$, respectively, is at most $\frac{2^{2 \lambda+1} \cdot q^{3}}{p}$.

Thus, for a statistical security parameter $n$, we can tolerate $\lambda=\log (p) / 2-3$ $\log (q)-n / 2$ bits of leakage. For space reasons, here we only can give a proof outline. The complete proof appears in the full version of this paper.

Proof Outline. For technical reasons, we will consider a setting where the generic bilinear group is extended with an additional oracle $\mathcal{O}_{D L}: \Xi \cup \Xi_{T} \rightarrow$ $\mathbb{Z}_{p} \cup \perp$, we will call this the extended generic bilinear group model. Intuitively, $\mathcal{O}_{D L}$ is an oracle for the discrete log problem, but only works on inputs that have not yet appeared since the oracles $\mathcal{O}, \mathcal{O}_{e}, \mathcal{O}_{T}$ have been initialized.

The proof outline is as follows. We will first show that the discrete logarithm problem (DL) is hard in the (base group of the) extended GBG model. We then give a reduction which shows how any adversary that can break the CCA1 security (without leakage) of BEG* in the (normal) GBG model, can solve the discrete log problem in the extended GBG model. Next, we extend this proof to get our main result, namely a reduction of the CCLA1 security of BEG ${ }^{*}$ to the discrete $\log$ problem.

CCA1 SECURITY OF BEG* . Let $\mathcal{F}$ be an adversary that can break the CCA1 security of BEG*. We construct an adversary $\mathcal{G}$ for DL (using $\mathcal{F}$ as a black-box) by letting $\mathcal{G}$ simulate the $\operatorname{Exp}_{\mathrm{BEG}}^{\text {cca1 }}(\mathcal{F}, p)$ experiment, where in this experiment $\mathcal{G}$ uses its DL challenge $\xi(y)$ as either the secret key $\xi(x)$ or the challenge encapsulated key $\xi(s)$ with probability $1 / 2$ respectively.

During the CCA1 experiment, $\mathcal{F}$ (which initially gets $\xi_{T}(x)$, and after the last decapsulation query gets $\xi(s))$ will learn the representation of elements $\xi_{T}\left(e_{1}\right)$, $\xi_{T}\left(e_{2}\right), \ldots$ from the target group. One can show that just from observing $\mathcal{F}$ 's oracle queries, $\mathcal{G}$ can assign to each $e_{i}$ an equation $e_{i}=a_{i}+b_{i} \cdot x+c_{i} \cdot s+d_{i} \cdot s^{2}$ where it knows the coefficients $a_{i}, b_{i}, c_{i}, d_{i} \in \mathbb{Z}_{p}$. Similarly, for representations $\xi\left(e_{1}\right), \xi\left(e_{2}\right), \ldots$ of elements in the base group that $\mathcal{F}$ learns, $\mathcal{G}$ can extract $a_{i}, b_{i}$ such that $e_{i}=a_{i}+b_{i} \cdot s$. To get an idea why this is the case, consider, e.g., the case where $\mathcal{F}$ makes a query $\xi_{T}(v \cdot w) \leftarrow \mathcal{O}_{e}(\xi(v), \xi(w))$. If $\xi(v)$ (same for $\xi(w)$ ) was never seen before, $\mathcal{G}$ first calls $\mathcal{O}_{D L}(\xi(v))$ to learn $v$. (Recall that $\mathcal{G}$ is in the extended GBG model.) Now $\mathcal{G}$ knows $a, b, a^{\prime}, b^{\prime}$ s.t. $v=a+b \cdot s$ and $w=a^{\prime}+b^{\prime} \cdot s$, which implies $v \cdot w=a^{\prime \prime}+b^{\prime \prime} \cdot x+c^{\prime \prime} \cdot s+d^{\prime \prime} \cdot s^{2}$ with $a^{\prime \prime}=a+a^{\prime}, b^{\prime \prime}=0, c^{\prime \prime}=$ $a \cdot b^{\prime}+a^{\prime} \cdot b, d^{\prime \prime}=b \cdot b^{\prime}$.

Recall that $\mathcal{F}$ 's goal is to distinguish the decapsulated key $\xi_{T}(x \cdot s)$ from a random element. If $\mathcal{F}$ has advantage $\epsilon$ in doing so, it actually must compute the element $\xi_{T}(x \cdot s)$ with probability $\epsilon$. Which means we learn $a, b, c, d$ such that

$$
x \cdot s=a+b \cdot x+c \cdot s+d \cdot s^{2},
$$

this can be solved for $s$ or $x$ (or both). Thus $\mathcal{G}$ will learn the discrete $\log$ of $\xi(y)$ with probability at least $\varepsilon / 2$ (as we initially randomly set $\xi(y)=s$ or $\xi(y)=x$ ).

CCLA1 SECURITY OF BEG*. The proof in the case of leakage attacks is more delicate. In a CCLA1 attack, with the $i$ th decapsulation query, the adversary $\mathcal{F}$ also 
learns the output of the leakage functions $f_{i}, g_{i}$. If we had no upper bound on the output length of those functions, then $f_{i}$ and $g_{i}$ could just leak $\xi\left(R_{i}\right)$ and $\xi\left(x-R_{i}\right)$ respectively, from which $\mathcal{F}$ then could first compute the secret key $\xi(x)$ and then $\xi_{T}(x \cdot s)$. In this case, the reduction $\mathcal{G}$ does not learn an equation of the form eq.(2), but only the trivial equality $x \cdot s=x \cdot s$. We will prove that if the leakage bound $\lambda \ll \log p / 2$, then the leakage functions will not leak any representation of an element to $\mathcal{F}$ that $\mathcal{F}$ could not efficiently compute itself.

To see this, let us first make the simplifying assumption that the leakage functions $f_{i}, g_{i}$ are not given access to the group oracles $\mathcal{O}, \mathcal{O}_{e}, \mathcal{O}_{T}$. Then all the leakage functions can try to do, is to leak some element they get as input. Consider any such element, say $\xi\left(R_{i}\right)$. As $\xi\left(R_{i}\right)$ is only given as input to $f_{i-1}$ and $f_{i}$, at most $2 \lambda$ bits about this element can leak. If $2 \lambda \ll \log p$, then $\mathcal{F}$ will have high min-entropy about $\xi\left(R_{i}\right)$ even given this $2 \lambda$ bits of leakage. Thus it is very unlikely that it can guess $\xi\left(R_{i}\right)$.

Now consider the general case, where the leakage functions can use the group oracles. Now the leakage functions can trivially leak the representation of some group element, say $f_{1}, f_{2}, \ldots$ all use $\mathcal{O}$ to compute $\xi(z)$ for some fixed $z$ and each leaks $\lambda$ bit of $\xi(z)$ until $\mathcal{F}$ learns the entire $\xi(a)$. Now $\mathcal{F}$ does get the representation of an element $\xi(a)$ without receiving it from the group oracles, but that is no problem, as $\mathcal{G}$ will know an $a, b$ such that $a+b \cdot s=z$ (namely $a=z$ and $b=0$ ), and that's all we care about.

Now the $f_{i}$ leakage function (similarly for $\left.g_{i}\right)$ can use their input $\xi\left(R_{i-1}\right)$ to compute elements $\xi(z)$ where $\mathcal{G}$ only knows $a, b$ (where $b \neq 0$ ) such that $z=$ $a+b \cdot r_{0}$. We call such a representation "bound" (as opposed to "free" representations $\xi(z)$ where $\mathcal{G}$ trivially learns $z$ by just observing $f_{i}$ 's oracle queries). It would be a problem if a bound representation could leak to $\mathcal{F}$. As said before, the $f_{i}$ 's can trivially leak $2 \lambda$ bits about a bound element, as, e.g., $f_{i-1}$ and $f_{i}$ have access to $\xi\left(R_{i}\right)$ (recall that $R_{i}=\sum_{j=0}^{i} r_{j}$ where each $r_{j}$ is uniformly random). But it is not clear how any other leakage function $f_{j}(j \notin\{i-1, i\})$ would compute the element $\xi\left(R_{i}\right)$ or any other element derived from it; since the sharings are randomized during each invocation, the values $\xi\left(R_{j-1}\right), r_{j}$ that $f_{j}$ has are completely independent of $R_{i}$ (and thus $\xi\left(R_{i}\right)$ ). In fact, we show that if $\mathcal{F}$ manages to choose leakage functions such that the same bound element is computed by $f_{i}$ and $f_{j}$ (where $j>i+1$ ) with probability $\epsilon$, then $\mathcal{F}$ can be used to solve the discrete logarithm problem with probability $\epsilon / 2^{2 \lambda} q$. The idea is to use the discrete logarithm challenge $\xi(y)$ as $\xi\left(r_{j}\right)$ for a random $j$. Note that to simulate the experiment, $\mathcal{G}$ only needs $\xi\left(r_{j}\right)$ not $r_{j}$, except to compute the $2 \lambda$ bits of leakage from the $j$ th decapsulation query. (As here the leakage functions $f_{j}, g_{j}$ expect $r_{j}$ as input.) We let $\mathcal{G}$ randomly guess this leakage, which will be correct with probability $2^{-2 \lambda}$. Now assume we have two identical bound elements $\xi(z)$ computed by $f_{i^{\prime}}$ and $f_{i^{\prime \prime}}$ where $i^{\prime \prime}>i^{\prime}+1$. As this query was made by $f_{i^{\prime}}$, and up to this point $\mathcal{G}$ only used $r_{0}, \ldots, r_{i^{\prime}}$ that it sampled himself, he will know $z$. As this query was also made by $i^{\prime \prime}, \mathcal{G}$ learns $a, b \neq 0$ such that $z=a+b \cdot r_{j}$, and thus can solve this equality to get $r_{j}$. 


\section{References}

1. Abdalla, M., Bellare, M., Rogaway, P.: The oracle Diffie-Hellman assumptions and an analysis of DHIES. In: Naccache, D. (ed.) CT-RSA 2001. LNCS, vol. 2020, p. 143. Springer, Heidelberg (2001)

2. Akavia, A., Goldwasser, S., Vaikuntanathan, V.: Simultaneous hardcore bits and cryptography against memory attacks. In: Reingold, O. (ed.) TCC 2009. LNCS, vol. 5444, pp. 474-495. Springer, Heidelberg (2009)

3. Alwen, J., Dodis, Y., Naor, M., Segev, G., Walfish, S., Wichs, D.: Public-key encryption in the bounded-retrieval model. In: Gilbert, H. (ed.) EUROCRYPT 2010. LNCS, vol. 6110, pp. 113-134. Springer, Heidelberg (2010)

4. Alwen, J., Dodis, Y., Wichs, D.: Leakage-resilient public-key cryptography in the bounded-retrieval model. In: Halevi, S. (ed.) CRYPTO 2009. LNCS, vol. 5677, pp. 36-54. Springer, Heidelberg (2009)

5. Barak, B., Shaltiel, R., Wigderson, A.: Computational analogues of entropy. In: RANDOM-APPROX, pp. 200-215 (2003)

6. Biham, E., Shamir, A.: Differential fault analysis of secret key cryptosystems. In: Kaliski Jr., B.S. (ed.) CRYPTO 1997. LNCS, vol. 1294, pp. 513-525. Springer, Heidelberg (1997)

7. Boneh, D., Boyen, X., Goh, E.-J.: Hierarchical identity based encryption with constant size ciphertext. In: Cramer, R. (ed.) EUROCRYPT 2005. LNCS, vol. 3494, pp. 440-456. Springer, Heidelberg (2005)

8. Boneh, D., De Millo, R.A., Lipton, R.J.: On the importance of checking cryptographic protocols for faults (extended abstract). In: Fumy, W. (ed.) EUROCRYPT 1997. LNCS, vol. 1233, pp. 37-51. Springer, Heidelberg (1997)

9. Brakerski, Z., Kalai, Y.T., Katz, J., Vaikuntanathan, V.: Overcoming the hole in the bucket: Public-key cryptography resilient to continual memory leakage. In: 51st FOCS. IEEE Computer Society Press, Los Alamitos (2010)

10. Cash, D., Ding, Y.Z., Dodis, Y., Lee, W., Lipton, R.J., Walfish, S.: Intrusion-resilient key exchange in the bounded retrieval model. In: Vadhan, S.P. (ed.) TCC 2007. LNCS, vol. 4392, pp. 479-498. Springer, Heidelberg (2007)

11. Chari, S., Jutla, C.S., Rao, J.R., Rohatgi, P.: Towards sound approaches to counteract power-analysis attacks. In: Wiener, M. (ed.) CRYPTO 1999. LNCS, vol. 1666, pp. 398-412. Springer, Heidelberg (1999)

12. Clavier, C., Joye, M.: Universal exponentiation algorithm. In: Koç, Ç.K., Naccache, D., Paar, C. (eds.) CHES 2001. LNCS, vol. 2162, pp. 300-308. Springer, Heidelberg (2001)

13. Di Crescenzo, G., Lipton, R.J., Walfish, S.: Perfectly secure password protocols in the bounded retrieval model. In: Halevi, S., Rabin, T. (eds.) TCC 2006. LNCS, vol. 3876, pp. 225-244. Springer, Heidelberg (2006)

14. Dodis, Y., Goldwasser, S., Kalai, Y.T., Peikert, C., Vaikuntanathan, V.: Publickey encryption schemes with auxiliary inputs. In: Micciancio, D. (ed.) Theory of Cryptography. LNCS, vol. 5978, pp. 361-381. Springer, Heidelberg (2010)

15. Dodis, Y., Haralambiev, K., Lopez-Alt, A., Wichs, D.: Cryptography against continuous memory attacks. In: 51st FOCS. IEEE Computer Society Press, Los Alamitos (2010)

16. Dodis, Y., Haralambiev, K., Lopez-Alt, A., Wichs, D.: Efficient public-key cryptography in the presence of key leakage. In: Abe, M. (ed.) ASIACRYPT 2010. LNCS, vol. 6477, pp. 595-612. Springer, Heidelberg (2010) 
17. Dodis, Y., Kalai, Y.T., Lovett, S.: On cryptography with auxiliary input. In: 41st ACM STOC. ACM Press, New York (2009)

18. Dodis, Y., Pietrzak, K.: Leakage-resilient pseudorandom functions and side-channel attacks on feistel networks. In: Rabin, T. (ed.) CRYPTO 2010. LNCS, vol. 6223, pp. 21-40. Springer, Heidelberg (2010)

19. Dziembowski, S.: Intrusion-resilience via the bounded-storage model. In: Halevi, S., Rabin, T. (eds.) TCC 2006. LNCS, vol. 3876, pp. 207-224. Springer, Heidelberg (2006)

20. Dziembowski, S.: On forward-secure storage (extended abstract). In: Dwork, C. (ed.) CRYPTO 2006. LNCS, vol. 4117, pp. 251-270. Springer, Heidelberg (2006)

21. Dziembowski, S., Maurer, U.M.: Optimal randomizer efficiency in the boundedstorage model. Journal of Cryptology 17(1), 5-26 (2004)

22. Dziembowski, S., Pietrzak, K.: Intrusion-resilient secret sharing. In: 48th FOCS, pp. 227-237. IEEE Computer Society Press, Los Alamitos (2007)

23. Dziembowski, S., Pietrzak, K.: Leakage-resilient cryptography. In: 49th FOCS, pp. 293-302. IEEE Computer Society Press, Los Alamitos (2008)

24. El Gamal, T.: On computing logarithms over finite fields. In: Williams, H.C. (ed.) CRYPTO 1985. LNCS, vol. 218, pp. 396-402. Springer, Heidelberg (1986)

25. Faust, S., Kiltz, E., Pietrzak, K., Rothblum, G.N.: Leakage-resilient signatures. In: Micciancio, D. (ed.) Theory of Cryptography. LNCS, vol. 5978, pp. 343-360. Springer, Heidelberg (2010)

26. Gandolfi, K., Mourtel, C., Olivier, F.: Electromagnetic analysis: Concrete results. In: Koç, Ç.K., Naccache, D., Paar, C. (eds.) CHES 2001. LNCS, vol. 2162, pp. 251261. Springer, Heidelberg (2001)

27. Goldwasser, S., Kalai, Y.T., Rothblum, G.N.: One-time programs. In: Wagner, D. (ed.) CRYPTO 2008. LNCS, vol. 5157, pp. 39-56. Springer, Heidelberg (2008)

28. Goldwasser, S., Rothblum, G.N.: Securing computation against continuous leakage. In: Rabin, T. (ed.) CRYPTO 2010. LNCS, vol. 6223, pp. 59-79. Springer, Heidelberg (2010)

29. Alex Halderman, J., Schoen, S.D., Heninger, N., Clarkson, W., Paul, W., Calandrino, J.A., Feldman, A.J., Appelbaum, J., Felten, E.W.: Lest we remember: coldboot attacks on encryption keys. ACM Commun. 52(5), 91-98 (2009)

30. Harnik, D., Naor, M.: On everlasting security in the hybrid bounded storage model. In: Bugliesi, M., Preneel, B., Sassone, V., Wegener, I. (eds.) ICALP 2006.Part II LNCS, vol. 4052, pp. 192-203. Springer, Heidelberg (2006)

31. Håstad, J., Impagliazzo, R., Levin, L.A., Luby, M.: A pseudorandom generator from any one-way function. SIAM Journal on Computing 28(4), 1364-1396 (1999)

32. IEEE P1363a Committee. IEEE P1363a / D9 - standard specifications for public key cryptography: Additional techniques (June 2001), http://grouper.ieee.org/groups/1363/index.html/draft Version 9

33. Juma, A., Vahlis, Y.: Protecting cryptographic keys against continual leakage. In: Rabin, T. (ed.) CRYPTO 2010. LNCS, vol. 6223, pp. 41-58. Springer, Heidelberg (2010)

34. Katz, J., Vaikuntanathan, V.: Signature schemes with bounded leakage resilience. In: Matsui, M. (ed.) ASIACRYPT 2009. LNCS, vol. 5912, pp. 703-720. Springer, Heidelberg (2009)

35. Koblitz, N., Menezes, A.J.: Another look at generic groups. Advances in Mathematics of Communications 1, 13-28 (2007)

36. Kocher, P.C.: Timing attacks on implementations of Diffie-Hellman, RSA, DSS, and other systems. In: Koblitz, N. (ed.) CRYPTO 1996. LNCS, vol. 1109, pp. 104-113. Springer, Heidelberg (1996) 
37. Kocher, P.C., Jaffe, J.: Leak-Resistant Cryptographic Method and Apparatus. United States Patent 6304658 B1 (October 16, 2001)

38. Kocher, P.C., Jaffe, J., Jun, B.: Differential power analysis. In: Wiener, M. (ed.) CRYPTO 1999. LNCS, vol. 1666, pp. 388-397. Springer, Heidelberg (1999)

39. Maurer, U.M.: A provably-secure strongly-randomized cipher. In: Damgård, I. (ed.) EUROCRYPT 1990. LNCS, vol. 473, pp. 361-373. Springer, Heidelberg (1991)

40. Micali, S., Reyzin, L.: Physically observable cryptography (extended abstract). In: Naor, M. (ed.) TCC 2004. LNCS, vol. 2951, pp. 278-296. Springer, Heidelberg (2004)

41. Naor, M., Segev, G.: Public-key cryptosystems resilient to key leakage. In: Halevi, S. (ed.) CRYPTO 2009. LNCS, vol. 5677, pp. 18-35. Springer, Heidelberg (2009)

42. Nechaev, V.I.: Complexity of a determinate algorithm for the discrete logarithm. Mathematical Notes 55(2), 165-172 (1994)

43. European Network of Excellence (ECRYPT). The side channel cryptanalysis lounge, http://www.crypto.ruhr-uni-bochum.de/en_sclounge.html (retrieved on March 29, 2008)

44. Pietrzak, K.: A leakage-resilient mode of operation. In: Joux, A. (ed.) EUROCRYPT. LNCS, pp. 462-482. Springer, Berlin (2009)

45. Quisquater, J.-J., Samyde, D.: Electromagnetic analysis (ema): Measures and counter-measures for smart cards. In: E-smart, pp. 200-210 (2001)

46. Quisquater, J.-J., Koene, F.: Side channel attacks: State of the art (October 2002) [43]

47. Rackoff, C., Simon, D.R.: Non-interactive zero-knowledge proof of knowledge and chosen ciphertext attack. In: Feigenbaum, J. (ed.) CRYPTO 1991. LNCS, vol. 576, pp. 433-444. Springer, Heidelberg (1992)

48. Certicom research, standards for efficient cryptography group (SECG) - sec 1: Elliptic curve cryptography (September 20, 2000), http://www.secg.org/secg_docs.htm version 1.0

49. Shoup, V.: Lower bounds for discrete logarithms and related problems. In: Fumy, W. (ed.) EUROCRYPT 1997. LNCS, vol. 1233, pp. 256-266. Springer, Heidelberg (1997)

50. Shoup, V.: ISO 18033-2: An emerging standard for public-key encryption (December 2004), http://shoup.net/iso/std6.pdf (final Committee Draft)

51. Standaert, F.-X., Pereira, O., Yu,Y., Quisquater, J.-J., Yung, M., Oswald, E.: Leakage resilient cryptography in practice. Cryptology ePrint Archive, Report 2009/341 (2009), http://eprint.iacr.org/

52. Trichina, E., Bellezza, A.: Implementation of elliptic curve cryptography with builtin counter measures against side channel attacks. In: Kaliski Jr., B.S., Koç, Ç.K., Paar, C. (eds.) CHES 2002. LNCS, vol. 2523, pp. 98-113. Springer, Heidelberg (2003)

53. Vadhan, S.P.: On constructing locally computable extractors and cryptosystems in the bounded storage model. In: Boneh, D. (ed.) CRYPTO 2003. LNCS, vol. 2729, pp. 61-77. Springer, Heidelberg (2003) 